\title{
New Bounds on the Number of Frictionless Fingers Required to Immobilize 2D Objects
}

\author{
Elon Rimon \\ Dept. of Mechanical Engineering \\ Technion, Israel Institute of Technology
}

\author{
Joel W. Burdick \\ Dept. of Mechanical Engineering \\ California Institute of Technology
}

\section{Introduction}

This paper develops new lower bounds on the number of frictionless fingers or fixtures which are required to immobilize planar objects. We study in detail the case of objects with smooth boundaries, and polygonal objects. Analogous results for the case of piecewise smooth objects follow directly from the analysis presented herein. These results have obvious applications to fixture planning and grasp planning $[2,4,8]$, as we show that it is possible to immobilize objects with fewer fingers than was previously thought possible.

The issue of how many frictionless contacts are required to immobilize an object has a lengthy history. Reuleaux (1875) [9] found that at least 4 frictionless contact points are required for force closure (which is one means to immobilize an object) of $2 \mathrm{D}$ objects. Somoff (1900) [14] found that at least 7 frictionless contact points are required for force closure of $3 \mathrm{D}$ objects. Much later, Markenscoff et. al (1990) [6] established that 4 or 7 contact points suffice to achieve force closure of almost any $2 \mathrm{D}$ or $3 \mathrm{D}$ object. The inscribed disc used by Markenscoff et. al will also be used in our derivations.

Recently, Czyzowicz et. al [5] showed that generic 2D and $3 \mathrm{D}$ polygonal objects can be immobilized respectively by 3 and 4 frictionless point contacts. In this paper, we show that generic smooth planar objects, and all polygonal objects, can be immobilized by 3 frictionless convex fingers. Thus we extend the results of [5] to a much larger class of objects. We are also able to overcome the limitation of the analysis in [5] to polygons without parallel edges. Further, if we are allowed to choose suitably concave fingers (which still contact the object at points), we show that generic smooth planar objects, and all polygonal objects, can be immobilized with 2 frictionless fingers. This possibility, to our knowledge, has never appeared in the literature before.

The new lower bounds are based on the inclusion of the curvature of the object and the finger surfaces into the analysis of the object's mobility. We developed a mobility theory which includes curvature effects in $[10,11]$. This mobility theory plays a central role in this work, and it is reviewed in Section 2. Next we describe relevant properties of the maximal inscribed disc. Sections 4 and 5 focus on the immobilization of smooth objects by 3 convex and 2 concave fingers. The last section extends the bounds to polygonal objects. Due to space limitation, several proofs have been omitted or reduced. They can be found in [12].

\section{C-Space Approach to Rigid Body Mobility}

The essential components of our mobility theory $[10,11]$ are now reviewed, as these concepts are the basis for our derivations. This analysis is concerned with the mobility of an object $\mathcal{B}$ held in point contact by $k$ stationary and frictionless finger bodies $\mathcal{A}_{1}, \ldots, \mathcal{A}_{k}$ in an equilibrium grasp. The bodies are either $2 \mathrm{D}$ or $3 \mathrm{D}$. The analysis is formulated in $\mathcal{B}$ 's configuration space. Let $q=(d, \theta)$ be a parametrization of $\mathcal{B}$ 's c-space in terms of hybrid coordinates, where $d \in \mathbb{R}^{3}$ directly parametrizes translation and $\theta \in \mathbb{R}^{3}$ parametrizes $S O(3)$ via the exponential map. $\widehat{\theta}=\theta /\|\theta\|$ is the axis of rotation and $\|\theta\|$ is the angle of rotation. We regard $S O(2)$ as a subgroup of $S O(3)$ with rotation axis $\hat{\theta}$ normal to the plane. Thus $\mathcal{B}$ 's configuration space is parametrized by $\mathbb{R}^{m}$, where $m=\frac{1}{2} n(n+1)(m=3$ or 6$)$.

Consider Fig. 1 , where $\mathcal{B}$ is an ellipse contacted by a finger $\mathcal{A}_{i}$. $\mathcal{B}$ 's configuration space (c-space) is $q=\left(d_{x}, d_{y}, \theta\right)$, and the c-space obstacle (c-obstacle) due to $\mathcal{A}_{i}$ is the set of configurations where $\mathcal{B}$ intersects the stationary "obstacle" $\mathcal{A}_{i}$ (Fig. 1(b)). Thus, if $q_{0}$ is $\mathcal{B}$ 's contact configuration, $q_{0}$ lies on the c-obstacle boundary, which is denoted $\mathcal{S}_{i}$. When $\mathcal{B}$ is contacted by $k$ fingers, $q_{0}$ lies on the intersection of $\mathcal{S}_{i}$ for $i=1, \ldots, k$.

\section{$2.11^{\text {st }}$ and $2^{\text {nd }}$ order free motions}

The free motions of $\mathcal{B}$ are those local motions of $\mathcal{B}$ along which it either breaks away from or roll-slides on the finger surfaces. Equivalently, the free motions of $\mathcal{B}$ at $q_{0}$ are the c-space paths that emanate from $q_{0}$ and locally lie in the freespace, which is the complement of the $c-$ obstacle interiors. Our mobility theory is based on the $1^{\text {st }}$ and $2^{\text {nd }}$ properties of the free motion curves. In the following $\hat{n}_{i}\left(q_{0}\right)$ denotes the outward pointing unit normal to $\mathcal{S}_{i}$ at $q_{0}$ (Fig. $\left.1(\mathrm{~b})\right)$.

Definition 1 ([10]) The $1^{\text {st }}$ order free motions of $\mathcal{B}$ at $q_{0}$ is the halfspace of $T_{q_{0}} \mathbb{R}^{m}$ satisfying $M_{i}^{1}\left(q_{0}\right) \triangleq\left\{\dot{q} \in T_{q_{0}} \mathbb{R}^{m}: \hat{\mathbf{n}}_{i}\left(q_{0}\right) \cdot \dot{q} \geq 0\right\}$. The halfspace's boundary, $T_{q_{0}} \mathcal{S}_{i}=\left\{\dot{q} \in T_{q_{0}} \mathbb{R}^{m}: \hat{\mathbf{n}}_{i}\left(q_{0}\right) \cdot \dot{q}=0\right\}$, 

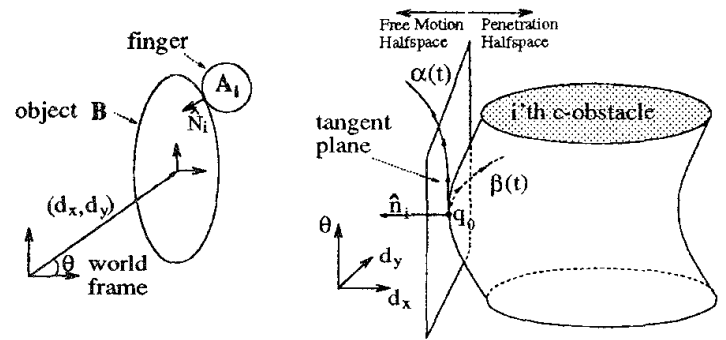

Figure 1. $1^{\text {st }}$ order approximation to the free motions of $\mathcal{B}$

is called the set of $1^{\text {st }}$ order roll-slide motions. Its interior, $\left\{\dot{q} \in T_{q_{0}} \mathbb{R}^{m}: \hat{\mathrm{n}}_{i}\left(q_{0}\right) \cdot \dot{q}>0\right\}$, is termed the set of $1^{\text {st }}$ order escape motions. For $k$ fingers, the set of $1^{\text {st }}$ order free motions is: $M_{1, \ldots, k}^{1}\left(q_{0}\right) \triangleq \cap_{i=1}^{k} M_{i}^{1}\left(q_{0}\right)$.

Thus, along escape motions $\mathcal{B}$ increases its distance from $\mathcal{A}_{i}$ to first order, which implies that it locally breaks away from $\mathcal{A}_{i} . \mathcal{B}$ keeps its distance from $\mathcal{A}_{i}$ zero to first order along $1^{\text {st }}$ order roll-slide motions, and it is not possible to determine from $1^{\text {st }}$ order considerations if $\mathcal{B}$ locally breaks away or penetrates $\mathcal{A}_{i}$. As we shall see, all the free motions of $\mathcal{B}$ at an equilibrium grasp are roll-slide to $1^{\text {st }}$ order. This important fact implies that the mobility of $\mathcal{B}$ at an equilibrium grasp depends on the $2^{\text {nd }}$ order properties of its local motions.

The $2^{\text {nd }}$-order geometry of the free-motion curves and the c-obstacle boundaries is determined by their curvature and curvature form, respectively. The curvature form of $\mathcal{S}_{i}$ at $q_{0} \in \mathcal{S}_{i}$ is denoted $\kappa_{i}\left(q_{0}, \dot{q}\right)$, where $\kappa_{i}\left(q_{0}, \dot{q}\right)=\dot{q}^{T}\left[D \hat{\mathrm{n}}_{i}\left(q_{0}\right)\right] \dot{q}$ for $\dot{q} \in T_{q_{0}} \mathcal{S}_{i}$. An explicit formula for the curvature form was derived in [10]. We show in [10] that the free-motion curves are determined to second-order by their velocity and acceleration at $q_{0}$.

Definition 2 ([10]) The $2^{\text {nd }}$ order free motions of $\mathcal{B}$ at $q_{0}$ is the subset of $(\dot{q}, \vec{q})$ satisfying $M_{i}^{2}\left(q_{0}\right) \triangleq\left\{(\dot{q}, \ddot{q}): \hat{\mathrm{n}}_{i}\left(q_{0}\right) \cdot \dot{q}=0\right.$ and $\dot{q}^{T}\left[D \hat{\mathrm{n}}_{i}\left(q_{0}\right)\right] \dot{q}+$ $\left.\hat{\mathrm{n}}_{i}\left(q_{0}\right) \cdot \ddot{q} \geq 0\right\}$. Analogous to the $1^{\text {st }}$ order case, pairs $(\dot{q}, \ddot{q})$ that satisfy $\hat{\mathrm{n}}_{i}\left(q_{0}\right) \cdot \dot{q}=0$ and $\dot{q}^{T}\left[D \hat{\mathrm{n}}_{i}\left(q_{0}\right)\right] \dot{q}+\hat{\mathrm{n}}_{i}\left(q_{0}\right)$. $\ddot{q}=0$ are called $2^{\text {nd }}$ order roll-slide motions, and the other pairs in $M_{i}^{2}\left(q_{0}\right)$ are termed $\mathbf{2}^{\text {nd }}$ order escape motions. For $k$ fingers, $M_{1, \ldots, k}^{2}\left(q_{0}\right) \triangleq \cap_{i=1}^{k} M_{i}^{2}\left(q_{0}\right)$.

Any $1^{\text {st }}$ order escape motion determines a free-motion curve. Our definition of $2^{\text {nd }}$ order free motions focuses on those curves which are roll-slide to $1^{\text {st }}$ order. We show in [10] that if $(\dot{q}, \ddot{q}) \in M_{i}^{2}\left(q_{0}\right)$ is a $2^{\text {nd }}$ order escape motion, any c-space path $\alpha(t)$ with $\alpha(0)=q_{0}, \dot{\alpha}(0)=\dot{q}$, $\ddot{\alpha}(0)=\ddot{q}$, locally lies in the freespace for $t \geq 0$. If $(\dot{q}, \ddot{q}) \in M_{i}^{2}\left(q_{0}\right)$ is a $2^{\text {nd }}$ order roll-slide motion, it is not possible to determine from $2^{\text {nd }}$ order considerations if $\alpha(t)$ locally lies in the freespace.

\subsection{Interpretation of the Free Motions}

This paper focuses on 2D objects, for which the free motions have appealing graphical interpretation.
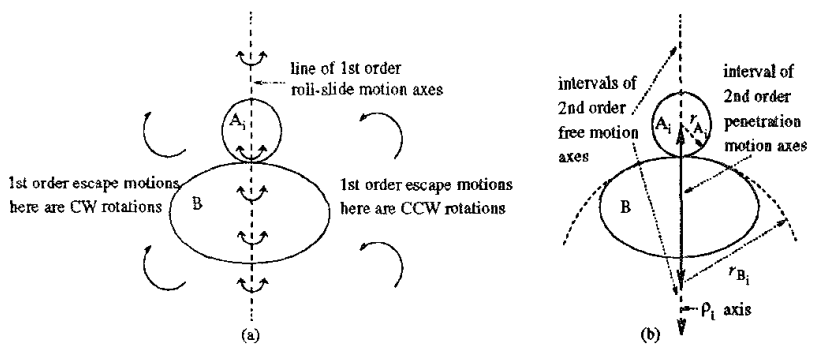

Figure 2. (a) $M_{i}^{1}\left(q_{0}\right)$ (b) $M_{i}^{2}\left(q_{0}\right)$ as instantaneous rotations

Graphical Interpretation of the $1^{\text {st }}$ Order Free Motions: If $q_{0}$ is $\mathcal{B}$ 's contact configuration with $\mathcal{A}_{i}$, the $1^{s t}$ order roll-slide motions of $\mathcal{B}$ are the hyperplane tangent to $\mathcal{S}_{i}$ at $q_{0} \in \mathcal{S}_{i}$ (Fig. 1). Let $l_{i}$ denote the line underlying the $i^{\text {th }}$ contact normal, and let $\rho_{i}$ be the distance along $l_{i}$ from the $i^{\text {th }}$ contact point, such that $\rho_{i}$ is positive on $\mathcal{B}$ 's side of the contact and negative on $\mathcal{A}_{i}$ 's side. It can be verified that the $1^{\text {st }}$ order roll-slide motions correspond to the instantaneous rotations of $\mathcal{B}$ about points of $l_{i}$ at a distance $\rho_{i}$, as $\rho_{i}$ sweeps $l_{i}$ from $-\infty$ to $\infty$. Note: Rotation about an axis "at infinity" gives pure translation in a direction perpendicular to $l_{i}$.

Formula for the curvature of a c-obstacle: In [10] we give a formula for the curvature of $\mathcal{S}_{i}, \kappa_{i}\left(q_{0}, \dot{q}\right)$, in terms of the curvatures of the boundary curves of $\mathcal{B}$ and $\mathcal{A}_{i}$ at their contact point. These curvatures are respectively denoted by the scalars $\kappa_{\mathcal{B}_{i}}$ and $\kappa_{\mathcal{A}_{\mathrm{i}}}$. We will need the specialization of the curvature formula for $\dot{q}$ which represent instantaneous rotations of $\mathcal{B}$ about points which lie along the line $l_{i}$. In the hybrid coordinates, tangent vectors are denoted $\dot{q}=(v, \omega)$, where $v$ is translational velocity and $\omega$ angular velocity. Instantaneous rotations of $\mathcal{B}$ thus have the form $\dot{q}=(0, \omega)$.

Lemma 2.1 ([13]) The curvature of $\mathcal{S}_{i}$ at $q_{0}$ along pure instantaneous rotation of $\mathcal{B}, \dot{q}=(0, \omega)$ such that $\|\omega\|=1$, about an axis at distance $\rho_{i}$ along $l_{i}$ is:

$\kappa_{i}\left(q_{0},(0, \omega)\right)=\left(\rho_{i} \kappa_{\mathcal{B}_{1}}-1\right)\left(\rho_{i} \kappa_{\mathcal{A}_{\mathbf{i}}}+1\right) /\left(\kappa_{\mathcal{A}_{\mathbf{i}}}+\kappa_{\mathcal{B}_{\mathbf{i}}}\right) .(1)$

Graphical Interpretation of $2^{\text {nd }}$ Order Free Motions: Formula (1) yields a graphical interpretation of the $2^{\text {nd }}$ order free motions. Let $r_{\mathcal{A}_{\mathrm{i}}}=1 / \kappa_{\mathcal{A}_{\mathrm{i}}}$ and $r_{\mathcal{B}_{i}}=1 / \kappa_{\mathcal{B}_{i}}$ be the radii of curvature of $\mathcal{A}_{i}$ and $\mathcal{B}$ at their contact point. Eq. (1) can be rewritten as:

$$
\kappa_{i}\left(q_{0},(0, \omega)\right)=\left(\rho_{i}-r_{\mathcal{B}_{\mathbf{i}}}\right)\left(\rho_{i}+r_{\mathcal{A}_{\mathrm{i}}}\right) /\left(\mathrm{r}_{\mathcal{A}_{\mathbf{i}}}+\mathrm{r}_{\mathcal{B}_{\mathbf{i}}}\right)
$$

The sign of $\kappa_{i}\left(q_{0},(0, \omega)\right)$ is thus solely a function of the relative magnitudes of $\rho_{i}, r_{\mathcal{B}_{\mathrm{i}}}$, and $r_{\mathcal{A}_{\mathrm{i}}}$. Consider Fig. 2(b) where $\mathcal{B}$ and $\mathcal{A}_{i}$ are convex at the contact point, hence $r_{\mathcal{B}_{i}}>0$ and $r_{\mathcal{A}_{i}}>0$. If $\rho_{i}<r_{\mathcal{B}_{i}}$ then $\mathcal{B}$ will penetrate $\mathcal{A}_{i}$ if this rotation, which is free to $1^{\text {st }}$ order, is attempted. If $\rho_{i}=r_{\mathcal{B}_{\mathrm{i}}}$, we get a $1^{\text {st }}$ order roll-slide motion that is also a $2^{\text {nd }}$ order roll-slide motion. If $\rho_{i}>r_{B_{i}}$ the bodies separate, and this is a $2^{\text {nd }}$ order escape motion. If the rotation axis is on the finger side of the contact, $\rho_{i}<0$, and a similar analysis holds with the role of $r_{\mathcal{A}_{i}}$ replacing the role of $r_{\mathcal{B}_{\mathrm{i}}}$. If one of the bodies, $\mathcal{B}$ say, is concave at the contact point, then $r_{\mathcal{B}_{i}}<0$. Also $r_{\mathcal{A}_{i}}+r_{\mathcal{B}_{i}} \leq 0$, since necessarily $r_{\mathcal{A}_{\mathrm{i}}} \leq\left|r_{\mathcal{B}_{\mathrm{i}}}\right|$. The $2^{\text {nd }}$ order free axes in this case lie between the centers of curvature of the two bodies, in $-\left|r_{\mathcal{B}_{\mathrm{i}}}\right| \leq \rho_{i} \leq-r_{\mathcal{A}_{\mathrm{i}}}$. 


\section{$2.3 \quad 1^{\text {st }}$ and $2^{\text {nd }}$ Order Mobility Indices}

The mobility indices are coordinate invariant integervalued functions that measure the mobility, or effective number of degrees of freedom, of $\mathcal{B}$ when it is held in an equilibrium-grasp configuration $q_{0}$. At an equilibrium grasp the net wrench (force and torque) on $\mathcal{B}$ must be zero. The wrench due to a normal contact force applied by $\mathcal{A}_{i}$ on $\mathcal{B}$ is a positive multiple of the finger c-obstacle normal $\hat{n}_{i}\left(q_{0}\right)[13]$. The equilibrium condition in c-space is thus characterized by the requirement that the origin be in the convex hull of the finger c-obstacles' normals. That is, there must exist scalars $\lambda_{1}, \ldots, \lambda_{k}$ such that

$$
0=\lambda_{1} \hat{\mathbf{n}}_{1}\left(q_{0}\right)+\cdots+\lambda_{\mathrm{k}} \hat{\mathbf{n}}_{k}\left(q_{0}\right) \text {, }
$$

where $\lambda_{i} \geq 0$ and $\sum_{i=1}^{k} \lambda_{i}=1$. We assume that each $\hat{\mathrm{n}}_{i}\left(q_{0}\right)$ in (3) is essential for the grasp, meaning that the origin cannot be positively spanned with any subcollection of $\left\{\hat{\mathbf{n}}_{1}\left(q_{0}\right), \ldots, \hat{\mathbf{n}}_{k}\left(q_{0}\right)\right\}$. At a $k$-finger equilibrium grasp $M_{1, \ldots, k}^{1}\left(q_{0}\right)$ forms a subspace, consisting of all the $1^{s t}$ order roll-slide motions with respect to each of the fingers. The dimension of this subspace is $m-k+1$ $(m=3$ or 6$)$, and it is defined as the $1^{\text {st }}$ order mobility index of the equilibrium grasp, $m_{q_{0}}^{1} \triangleq m-k+1$. $m_{q_{0}}^{1}$ is coordinate invariant [10]. However, $m_{q_{0}}^{1}$ is identical for all $k$-fingered grasps. This lack of discriminating power is remedied with our novel $2^{\text {nd }}$ order index which is based on the c-space curvature of $\mathcal{S}_{i}, \kappa_{i}\left(q_{0}, \dot{q}\right)$ Consider the $\lambda_{i}$ 's in the equilibrium condition (3). It is shown in [11] that the weighted sum of the c-obstacle curvature forms has a coordinate invariant structure which characterizes the $2^{n d}$ order mobility of $\mathcal{B}$.

Definition 3 ([11]) Let $\lambda_{1}, \ldots, \lambda_{k}$ be the coefficients of the equilibrium equation (3). The c-space relative curvature form for the equilibrium grasp is $\kappa_{\mathrm{rel}}\left(q_{0}, \dot{q}\right) \triangleq \sum_{i=1}^{k} \lambda_{\mathrm{i}} \kappa_{i}\left(q_{0}, \dot{q}\right)$, where $\dot{q} \in M_{1, \ldots, k}^{1}\left(q_{0}\right)$.

The $2^{\text {nd }}$ order mobility index of the equilibrium grasp, denoted $m_{q_{0}}^{2}$, is the number of non-negative eigenvalues of the quadratic form $\kappa_{\text {rel }}\left(q_{0}, \dot{q}\right)$.

By definition, $m_{q_{0}}^{1}$ is an upper bound on the values of $m_{q_{0}}^{2}$ i.e., $0 \leq m_{q_{0}}^{2} \leq m_{q_{0}}^{1}$. This inequality has an important interpretation: $2^{\text {nd }}$ order effects always reduce the mobility of the grasped object. We will use this effect to lower the bounds of the number of fingers necessary for immobilization. If $m_{q_{0}}^{1}=0$, the body is completely immobilized, and the $2^{\text {nd }}$ order index carries no useful information. If $\mathcal{B}$ is not immobilized to $1^{\text {st }}$ order $\left(m_{q_{o}}^{1}>0\right)$, it may be immobilized to $2^{\text {nd }}$ order $\left(m_{q_{0}}^{2}=0\right)$. A key interpretation of the $2^{n d}$ order index is provided in [11]: if $m_{q_{0}}^{2}=0$, any local motion of $\mathcal{B}$ is either $1^{\text {st }}$ order penetration, or it is $1^{\text {st }}$ order roll-slide and then it is necessarily $2^{\text {nd }}$ order penetration. Thus $m_{q_{0}}^{2}=0$ implies that $\mathcal{B}$ is completely immobilized.

\section{The Maximally Inscribed Disc}

The locally maximal inscribed disc will be a key tool in the derivations. This section discusses its properties using the non-smooth analysis theory of Clarke [3]. This theory deals with the derivative of scalar-valued functions $f$ which are not necessarily differentiable, but admit a generalized gradient, denoted $\partial f(x)$. This $\partial f(x)$ is a set of vectors that reduces to the classical gradient where $f$ is differentiable. See [3] for more details.

Let $\mathcal{B} \subset \mathbb{R}^{n}$ be a compact set with non-empty interior and smooth boundary, which is denoted bdy $(\mathcal{B})$. Consider the minimal distance of a point $x \in \mathcal{B}$ from bdy $(\mathcal{B})$ i.e., the function $\delta: \mathcal{B} \rightarrow \mathbb{R}$ defined by

$$
\delta(x) \triangleq \min _{y \in \operatorname{bdy}(\mathcal{B})}\{\|x-y\|\}
$$

The distance of a point from a set is a continuous function. Since $\mathcal{B}$ is compact, $\delta$ attains at least one maximum on $\mathcal{B}$. Intuitively, every local maximum $x$ of $\delta$ is the center of a maximally inscribed n-disc-a disc which lies wholly inside $\mathcal{B}$ whose radius is maximal with respect to all the disc-centers in a neighborhood of $x$.

We now establish that the local maxima of $\delta$ are feasible equilibrium grasps. The inward pointing unit normal to $\operatorname{bdy}(\mathcal{B})$ at $y \in \operatorname{bdy}(\mathcal{B})$ is denoted $\hat{N}(y)$. $\mathcal{I}(x)$ denotes the set of all points $y \in$ bdy $(\mathcal{B})$ at which $\delta(x)$ attains its value i.e., $\delta(x)=\|x-y\|$ for all $y \in \mathcal{I}(x)$.

Lemma 3.1 ([12]) Let $x$ be a local maximum of $\delta$. Then all the normals $\hat{N}(y)$ for $y \in \mathcal{I}(x)$ point toward $x$. Moreover, the convex hull of these normals, denoted co, contains the origin, $0 \in \operatorname{co}\{\hat{N}(y): y \in \mathcal{I}(x)\}$.

Sketch of proof: If $\delta$ is differentiable at an interior point $x$, then according to [3, Proposition 2.5.4] $x$ must have a unique closest point $y$ on $b \operatorname{by}(\mathcal{B})$ and $\nabla \delta(x)=(x-y) /\|x-y\|$. If $\delta$ is not differentiable at $x$, then according to [3, Theorem 2.5.1] the generalized gradient, $\partial \delta(x)$, is the convex hull of the unit vectors $(x-y) /\|x-y\|$ for all $y \in \mathcal{I}(x)$. Next we show in [12] that the vectors $(x-y) /\|x-y\|$ for $y \in \mathcal{I}(x)$ satisfy $(x-y) /\|x-y\|=\hat{N}(y)$. Hence the unit normals point toward $x$ and $\partial \delta(x)$ is:

$$
\partial \delta(x)=\operatorname{co}\{\hat{N}(y): \text { for all } y \in \mathcal{I}(x)\} .
$$

Let $x$ be now a local maximum of $\delta$. According to [3, Proposition 2.3.2], a necessary condition for $x$ to be a local maximum of $\delta$ is that zero be in $\partial \delta(x)$. This, with (4), imply the condition of the lemma.

Next we show that if $x$ is a local maximum of $\delta$, the points $y \in \mathcal{I}(x)$ determine an equilibrium grasp using normal finger forces. We will need Carathéodory's theorem [1]: if $\mathcal{X} \subset \mathbb{R}^{n}$ and $p \in \operatorname{co}\{\mathcal{X}\}$, then $p \in$ co $\{\mathcal{Y}\}$ for a finite subset $\mathcal{Y} \subset \mathcal{X}^{\prime}$ of $k \leq n+1$ points.

Corollary 3.2 The local maxima of $\delta$ determine feasible equilibrium grasps of $\mathcal{B}$.

Proof: At an equilibrium grasp the net wrench on $\mathcal{B}$ is zero. Since $x$ is a local maximum of $\delta$, we have from Lemma 3.1 that $0 \in\{\hat{N}(y): y \in \mathcal{I}(x)\}$. If $\mathcal{I}(x)$ is infinite, the origin lies in the convex hull of a finite subset of unit normals according to Carathéodory's theorem. Thus there exist $k$ unit normals such that $0=\lambda_{1} \hat{N}\left(y_{1}\right)+\cdots+$ $\lambda_{\mathrm{k}} \hat{N}\left(y_{k}\right)$, where $\lambda_{i} \geq 0$ and $\sum_{i=1}^{k} \lambda_{i}=1$. Application of normal finger forces of magnitudes $\lambda_{1}, \ldots, \lambda_{k}$ at $y_{1}, \ldots, y_{k}$ yields zero net force. Since the lines underlying each $\hat{N}\left(y_{i}\right)$ passes through $x$, each finger force generates zero torque about $x$. 
The following lemma (proven in [12]) asserts that the locally maximal inscribed disc generically touches the boundary of $\mathcal{B}$ at $k=2,3$ points when $\mathcal{B}$ is $2 \mathrm{D}$.

Lemma 3.3 ([12]) Let $x$ be local maximum of $\delta$. Then $\mathcal{I}(x)$ is generically finite and of size $k \leq n+1$.

In 2D the lemma can be interpreted in terms of the Voronoi diagram of a set $\mathcal{S}$. The vertices of the Voronoi diagram are equidistant from at least 3 points of $\mathcal{S}$. The lemma says that for generic sets none of these vertices is equidistant from 4 points. The next lemma follows from the fact that the inscribed disc lies inside $\mathcal{B}$.

Lemma 3.4 Let $\mathcal{B}$ be planar and let $x$ be a local maximum of $\delta$. Then the radius of the inscribed disc, $\delta(x)$, $i s$ a lower bound on the radius of curvature of bdy $(\mathcal{B})$ at the points $y \in \mathcal{I}(x)$ where bdy $(\mathcal{B})$ is convex. The bound is strict whenever $y \in \mathcal{I}(x)$ is an isolated point.

\section{Immobilizing Objects with 3 Convex Fingers}

We consider only generic smooth objects, whose inscribed disc touches bdy $(\mathcal{B})$ at $k=2$ or 3 points. Special objects for which $k$ is finite can be reduced to $k=2$ or 3 using Carathéodory's theorem. Research in progress suggests that special objects for which $k$ is infinite can also be immobilized with 3 convex fingers, except when their boundary consists of concentric circles.

We first show that if the maximal inscribed disc touches $\operatorname{bdy}(\mathcal{B})$ at 3 points, these points define an immobilizing equilibrium grasp. Next we show that if the maximal inscribed disc touches $b \operatorname{dy}(\mathcal{B})$ at 2 points, these points determine a nearby 3 -point immobilizing equilibrium grasp. We will write $M_{q_{0}}^{1}$ for $M_{1, \ldots, k}^{1}\left(q_{0}\right)$.

Proposition 4.1 ([12]) Let $\mathcal{B}$ be $2 D$, smooth, with non-empty interior. If a locally maximal inscribed disc touches bdy $(\mathcal{B})$ at three isolated points, $\mathcal{B}$ is completely immobilized by placing 9 convex fingers at these points (Fig. 3).

Sketch of proof: According to Corollary 3.2, an equilibrium grasp is obtained by placing 3 fingers at the three points. Using the mobility theory, $m_{q_{0}}^{1}=1$ for the grasp. Thus the $1^{\text {st }}$ order free motions of $\mathcal{B}$ lie on the one-dimensional subspace, $M_{q_{0}}^{1}$, of instantaneous rotations of $\mathcal{B}$ about the inscribed disc center $x$. Next we show that $m_{q_{0}}^{2}=0$, which implies complete immobility. We have to show that $\kappa_{\text {rel }}\left(q_{0}, \dot{q}\right)=\sum_{i=1}^{3} \lambda_{i} \kappa_{i}\left(q_{0}, \dot{q}\right)<0$ for all $\dot{q} \in M_{q_{0}}^{1}$. In terms of eq. (2), $x$ is located at $\rho_{i}=\delta(x)$ for $i=1,2,3$. There are two cases to consider. If $\mathcal{B}$ is convex at the $i^{\text {th }}$ contact point, then $\delta(x)<r_{\mathcal{B}_{i}}$ according to Lemma 3.4. Using (2), this implies that $\kappa_{i}\left(q_{0},(0, \omega)\right)<0$. If $\mathcal{B}$ is concave at the $i^{t h}$ contact, then according to (2) the interval of $2^{\text {nd }}$ order free axes lies on $\mathcal{A}_{i}$ 's side of the contact point. But $x$ lies on $\mathcal{B}$ 's side. Hence $\kappa_{i}\left(q_{0},(0, \omega)\right)<0$ in this case too.

Example: In the 3-finger grasp of Fig. $3, m_{q_{0}}^{1}=1$. However, $m_{q_{0}}^{2}=0$ for the grasp and the object is completely immobilized by the three fingers or fixtures.

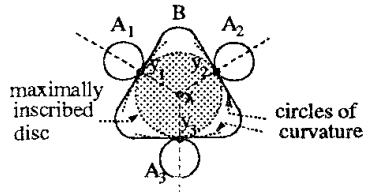

Figure 3. A 3-finger or 3-fixture immobilizing grasp

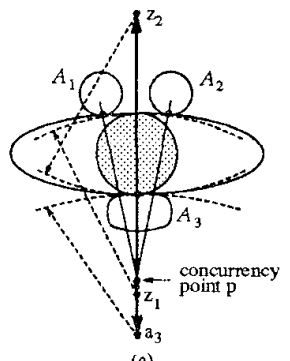

(a)

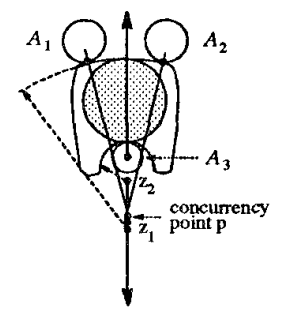

(b)
Figure 4. 3-finger immobilization starting at two points. Solid lines are $2^{\text {nd }}$ order penetration axes.

Note that the curvature of the fingers played no role in the last proof. Only the contact points location mattered. However, the fingers' curvature is important in the other generic case, where the inscribed disc touches bdy $(\mathcal{B})$ at two points. In this case our goal is to show that there exists a nearby 3 -finger immobilizing grasp.

We begin with a lemma that allows a local "splitting" of one of the contact points, $y_{1}$ say, into a pair $y_{11}, y_{12}$ located on either sides of $y_{1}$ (Fig. 4). The lemma further asserts that the contact point opposing the splitted pair can be moved slightly to generate a 3 -point equilibrium grasp. In the following, the lines underlying $\hat{N}\left(y_{11}\right)$ and $\hat{N}\left(y_{12}\right)$ are denoted $l_{11}$ and $l_{12}$. The center of curvature of $\operatorname{bdy}(\mathcal{B})$ at $y_{i}$ is denoted $z_{i}$.

Lemma 4.2 ([12]) Let a locally maximal inscribed disc touch bdy $(\mathcal{B})$ at two points $y_{1}$ and $y_{2}$. If the circles of curvature of $\operatorname{bdy}(\mathcal{B})$ at $y_{1}$ and $y_{2}$ are nonconcentric $^{1}$ i.e., $z_{1} \neq z_{2}$, there exist three points $y_{11}, y_{12}, y_{3} \in \operatorname{bdy}(\mathcal{B})$ such that: 1) The lines underlying $\hat{N}\left(y_{11}\right), \hat{N}\left(y_{12}\right), \hat{N}\left(y_{3}\right)$ are concurrent at a point $p$, and 2) The normals $\hat{N}\left(y_{11}\right), \hat{N}\left(y_{12}\right), \hat{N}\left(y_{3}\right)$ positively span the origin such that no two of them are collinear.

Thus, placing 3 fingers at $y_{11}, y_{12}, y_{3}$ gives an equilibrium grasp. However, for immobilization the finger opposing the splitted pair must be sufficiently flat.

Proposition 4.3 ([12]) Let $\mathcal{B}$ be $2 D$, smooth, with non-empty interior. Let a locally maximal inscribed disc touch $\mathrm{bdy}(\mathcal{B})$ at two isolated points $y_{1}$ and $y_{2}$. If the circles of curvature of bdy $(\mathcal{B})$ at $y_{1}$ and $y_{2}$ are nonconcentric, there exist three points on bdy $(\mathcal{B})$ such that $\mathcal{B}$ is completely immobilized by placing sufficiently flat convex fingers at these points.

Sketch of proof: Using Lemma 4.2 , we split $y_{1}$ into $y_{11}$ and $y_{12}$, and shift $y_{2}$ to $y_{3}$. An equilibrium grasp is

\footnotetext{
${ }^{1}$ Zero curvature at $y_{1}$ and $y_{2}$ is considered as $z_{1}=z_{2}=\{\infty\}$, and is excluded. This special case is considered in Prop. 4.4.
} 
obtained by placing 3 convex fingers $\mathcal{A}_{1}, \mathcal{A}_{2}, \mathcal{A}_{3}$ at the points $y_{11}, y_{12}, y_{3}$. The resulting grasp has $m_{q_{0}}^{1}=1$, and $M_{q_{0}}^{1}$ consists of instantaneous rotations of $\mathcal{B}$ about the concurrency point $p$. Next we show that $m_{q_{0}}^{2}=0$, which implies complete immobility. To show that $\kappa_{\text {rel }}\left(q_{0}, \dot{q}\right)=$ $\sum_{i=1}^{3} \lambda_{i} \kappa_{i}\left(q_{0}, \dot{q}\right)<0$ for all $\dot{q} \in M_{q_{0}}^{1}$, we will show that $\kappa_{1}\left(q_{0}, \dot{q}\right)$ and $\kappa_{2}\left(q_{0}, \dot{q}\right)$ can be made arbitrarily small, while $\kappa_{3}\left(q_{0}, \dot{q}\right)$ is strictly negative.

As $y_{11}$ and $y_{12}$ approach $y_{1}$ from both sides, the center of curvature of bdy $(\mathcal{B})$ at $y_{1 j}$ approaches $z_{1}$, and the intersection point of the lines $l_{11}$ and $l_{12}, p$, also approaches $z_{1}$. Hence the distance of $p$ from $y_{1 j}$, denoted $\rho_{j}$, approaches $r_{\mathcal{B}_{1 \mathrm{j}}}$, the radius of curvature of $\operatorname{bdy}(\mathcal{B})$ at $y_{1 j}$. As (2) contains a factor $\left(\rho_{j}-r_{\mathcal{B}_{1 j}}\right), \kappa_{j}\left(q_{0}, \dot{q}\right)$ can be made arbitrarily small for a local splitting of $y_{1}$.

Next we verify that $\kappa_{3}\left(q_{0}, \dot{q}\right)$ is strictly negative for instantaneous rotations about $p$. Let $\kappa_{\mathcal{B}}\left(y_{1}\right)$ and $\kappa_{\mathcal{B}}\left(y_{2}\right)$ be the curvatures of $\operatorname{bdy}(\mathcal{B})$ at $y_{1}$ and $y_{2}$. We may always assume that $\kappa_{\mathcal{B}}\left(y_{1}\right)>0$ [12]. Next we invoke a continuity argument, which allows us to determine the sign of $\kappa_{3}\left(q_{0}, \dot{q}\right)$ for $y_{3}=y_{2}$, and for instantaneous rotation of $\mathcal{B}$ about $p=z_{1}$. First consider the case where $\kappa_{\mathcal{B}}\left(y_{2}\right) \geq 0$ (Fig. 4(a)). Let $a_{3}$ be the center of curvature of the convex finger $\mathcal{A}_{3}$. According to (2), the interval of $2^{\text {nd }}$ order penetration axes lie between $z_{2}$ and $a_{3}$. According to Lemma 3.4, $r_{\mathcal{B}_{1}}>\delta(x)$ and $r_{\mathcal{B}_{2}}>\delta(x)$. Hence $z_{1}$ lies beyond $z_{2}$. By choosing $\mathcal{A}_{3}$ sufficiently flat at $y_{2}, a_{3}$ lies sufficiently far from $y_{2}$ so that $z_{1}$ lies between $z_{2}$ and $a_{3}$. Using (2), this implies that $\kappa_{3}\left(q_{0}, \dot{q}\right)<0$ for instantaneous rotation of $\mathcal{B}$ about $p=z_{1}$. If $\kappa_{\mathcal{B}}\left(y_{2}\right)<0$ (Fig. 4(b)), the interval of $2^{\text {nd }}$ order free axes lies between $z_{2}$ and $a_{3}$ on the finger side of the contact. As $z_{1} \neq z_{2}$ by hypothesis, for a sufficiently flat $\mathcal{A}_{3}$ the point $z_{1}$ lies outside the interval $\left[z_{2}, a_{3}\right]$. Hence $\kappa_{3}\left(q_{0}, \dot{q}\right)<0$ in this case too.

The two propositions lead to the following theorem.

Theorem 1 All generic smooth $2 D$ bodies can be immobilized by three frictionless convex fingers, provided that the fingers are sufficiently flat at the contact points.

Sketch a proof: According to Lemma 3.3, a maximally inscribed disc generically touches $\mathrm{bdy}(\mathcal{B})$ at $k=2$ or 3 isolated points. If $k=3$ we invoke Prop. 4.1. If $k=2$ we may invoke Prop. 4.3, provided that the circles of curvature of $\operatorname{bdy}(\mathcal{B})$ at $y_{1}$ and $y_{2}$ are non-concentric. The concentricity can be shown to be non-generic.

Special case Convex 2D Body with Parallel Edges: This special case commonly occurs in manmade objects. Thus we describe a 3 -finger immobilization scheme which starts from two parallel edges.

Proposition $4.4([12])$ Let $\mathcal{B}$ be $2 D$, smooth, with two parallel edges $e_{1}$ and $e_{2}$. Let a locally maximal inscribed disc touch bdy $(\mathcal{B})$ at two points $y_{1}$ and $y_{2}$ in the interior of $e_{1}$ and $e_{2}$. Then there exist three points on $\operatorname{bdy}(\mathcal{B})$ such that $\mathcal{B}$ is completely immobilized by placing sufficiently flat convex fingers at these points.

Sketch of proof: As depicted in Fig. 5, we first shift the endpoints of $e_{1}$ beyond the edge's limits, to obtain two contact points $y_{11}$ and $y_{12}$. Then we locate a third

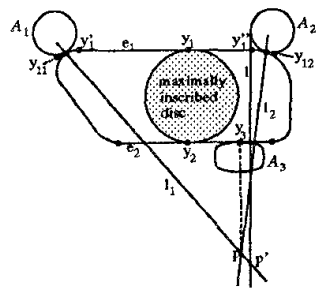

Figure 5. 3-finger immobilization starting at parallel edges

point, $y_{3}$, along $e_{2}$ to obtain a 3-point equilibrium grasp. The immobilization is obtained by exploiting the flatness of $\operatorname{bdy}(\mathcal{B})$ at $y_{3}$. See [12] for the full details.

\section{Immobilizing with 2 Concave Fingers}

We now show that 2 suitably curved frictionless fingers suffice to immobilize generic smooth objects.

Proposition 5.1 ([12]) Let $\mathcal{B}$ be a smooth $2 D$ shape, and let $y_{1}, y_{2} \in$ bdy $(\mathcal{B})$ form a 2-point equilibrium grasp of $\mathcal{B}$. If the circles of curvature of bdy $(\mathcal{B})$ at $y_{1}$ and $y_{2}$ are non-concentric, $\mathcal{B}$ is completely immobilized by placing two suitably curved fingers at these points.

The non-concentricity assumption excludes the possibility that $y_{1}$ and $y_{2}$ lie on two parallel edges of $\mathcal{B}$. However, we address this issue in Lemma 5.2 below.

Sketch of proof: At a 2-point equilibrium grasp the contact normals are antipodal and lie along a common line which is denoted $l$. The subspace $M_{q_{0}}^{1}$ is 2dimensicnal i.e., $m_{q_{0}}^{1}=2$. Thus $M_{q_{0}}^{1}$ consists of instantaneous rotations of $\mathcal{B}$ about an axis that sweeps $l$ from $-\infty$ to $\infty$. We will show that suitably curved fingers yield $m_{q_{0}}^{2}=0$ for the grasp.

The equilibrium condition (3), $\lambda_{1} \hat{n}_{1}\left(q_{0}\right)+\lambda_{2} \hat{n}_{2}\left(q_{0}\right)=$ 0 , implies that $\lambda_{1}=\lambda_{2}=1 / 2$. Hence it suffices to show that $2 \kappa_{\text {rel }}\left(q_{0}, \dot{q}\right)=\kappa_{1}\left(q_{0}, \dot{q}\right)+\kappa_{2}\left(q_{0}, \dot{q}\right)<0$ for all $\dot{q} \in$ $M_{q_{0}}^{1}$. We consider here only the case where $\mathcal{B}$ has nonzero curvature at both endpoints. We choose a finger $\mathcal{A}_{i}$ whose curvature at $y_{i}$ is $\epsilon$-away from the negated curvature of $\mathcal{B}$ at $y_{i}$, for $i=1,2$. More precisely, when $\mathcal{B}$ is strictly convex at $y_{i}$ i.e., $r_{\mathcal{B}_{\mathrm{i}}}>0$, we put a concave finger at $y_{i}$ such that $r_{\mathcal{A}_{i}}=\left(-r_{\mathcal{B}_{i}}\right)-\epsilon<0$ (Fig. $\left.6(\mathrm{a})\right)$. When $\mathcal{B}$ is strictly concave at $y_{i}$ i.e., $r_{B_{i}}<0$, we put a convex finger $\mathcal{A}_{i}$ such that $r_{\mathcal{A}_{i}}=\left(-r_{\mathcal{B}_{i}}\right)-\epsilon>0$ (Fig. $6(\mathrm{~b}))$. Note that in this case $\epsilon$ must satisfy $\epsilon<\left|\mathrm{r}_{\mathcal{B}_{\mathrm{i}}}\right|$.

Next we express $\rho_{1}$ and $\rho_{2}$ in terms of a common variable $\rho$. Let $\rho$ be the distance along $l$, measured from the midpoint of the contact points, such that $\rho$ is negative toward $y_{1}$ and positive toward $y_{2}$. Let $2 L$ be the distance between the contact points. Then $\rho_{1}=L+\rho$ and $\rho_{2}=L-\rho$. Substituting for $r_{\mathcal{A}_{\mathfrak{i}}}, \rho_{1}$, and $\rho_{2}$ in (2), then adding $\kappa_{1}\left(q_{0},(0, \omega)\right)$ to $\kappa_{2}\left(q_{0},(0, \omega)\right)$, gives a quadratic polynomial in $\rho$. The maximum value attained by this polynomial is $-\left\{\frac{1}{2}\left(\rho\left(z_{1}\right)-\rho\left(z_{2}\right)\right)^{2}+\epsilon\left(\rho\left(z_{1}\right)-\rho\left(z_{2}\right)\right)\right\}$. Where $z_{i}$ is the center of curvature of bdy $(\mathcal{B})$ at $y_{i}$, and $\rho\left(z_{i}\right)$ is the $\rho$ coordinate of $z_{i}$, for $i=1,2$. It follows that a sufficient condition for $\kappa_{\text {rel }}\left(q_{0},(0, \omega)\right)$ to be negative for all $\rho$ is $\epsilon<\frac{1}{2}\left|\rho\left(z_{1}\right)-\rho\left(z_{2}\right)\right|=\frac{1}{2}\left\|z_{1}-z_{2}\right\|$. To summarize, if the fingers' curvature is chosen according 

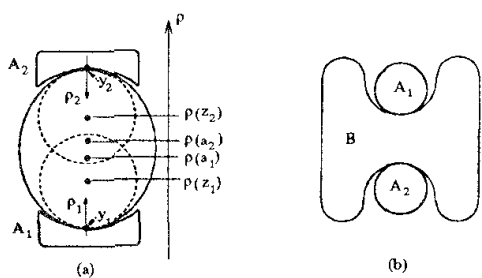

(b)

Figure 6. 2-finger immobilization exploiting concavities

to the rule $r_{\mathcal{A}_{\mathbf{i}}}=\left(-r_{\mathcal{B}_{\mathbf{i}}}\right)-\epsilon$ such that $\epsilon<\left|r_{\mathcal{B}_{\mathbf{i}}}\right|$ and $\epsilon<\frac{1}{2}\left\|z_{1}-z_{2}\right\|, m_{q_{0}}^{2}=0$ for the grasp (Fig. 6(a)).

Example: In Fig. 6(b) a $2 \mathrm{D}$ object is grasped or fixtured by two discs. $m_{q_{0}}^{1}=2$ for this grasp. Because of the concavity at the contact points, $\mathcal{B}$ is immobilized to $2^{\text {nd }}$ order i.e., $m_{q_{0}}^{2}=0$.

Special case--2D Body with Parallel Edges: As in the previous section, we consider the important special case where $\mathcal{B}$ has two parallel edges. The following lemma (proved in [12]) implies that 2-point grasps which do not lie on parallel edges always exist.

Lemma 5.2 ([12]) Any 2D smooth and compact body $\mathcal{B}$ possesses a 2-point equilibrium grasp such that the grasp points do not lie on parallel edges.

The following theorem is a corollary of Proposition 5.1.

Theorem 2 All generic smooth $2 D$ bodies can be immobilized by two, possibly concave, frictionless fingers.

The genericity assertion refers to the condition made in Prop. 5.1, that the object's circles of curvatures at the grasp points be non-concentric. This concentricity can be shown to be non-generic. Moreover, Lemma 5.2 guarantees that objects with parallel edges (whose circles of curvature are concentric at infinity), possess grasp points which do not lie on parallel edges. Hence almost all $2 \mathrm{D}$ objects with parallel edges also fall within the class of immobilizable objects of the theorem.

\section{Immobilizing Polygonal Objects}

This section summarizes work in [12], where the bounds derived for smooth objects are extended to polygons. First we characterize the c-obstacle of a finger contacting a polygon, then we derive the bounds.

\subsection{The C-Space Obstacles of a Polygon}

We now describe the finger c-obstacle when $\mathcal{A}_{i}$ contacts $\mathcal{B}$ at a vertex of $\mathcal{B}$. First consider the case where the contact occurs at a convex corner of $\mathcal{B}$ (Fig. $7($ a)). Assuming $\mathcal{A}_{i}$ is smooth, the contact normal between $\mathcal{A}_{i}$ and $\mathcal{B}$ is defined as the unique normal to $\operatorname{bdy}\left(\mathcal{A}_{i}\right)$ at the contact point. Note that a given $\mathcal{A}_{i}$ can contact $\mathcal{B}$ at the vertex as to generate any contact normal which is a convex combination of $\mathcal{B}$ 's edge normals. However, once the finger is placed, the contact normal is unique. The c-obstacle boundary is locally smooth at all of $\mathcal{B}$ 's configurations where its contact normal with $\mathcal{A}_{i}$ does not coincide with $\mathcal{B}$ 's edge normals. In fact, the curvature of the c-obstacle boundary is obtained by substituting $r_{\mathcal{B}_{i}}=0$ in the curvature formula $(2)$.
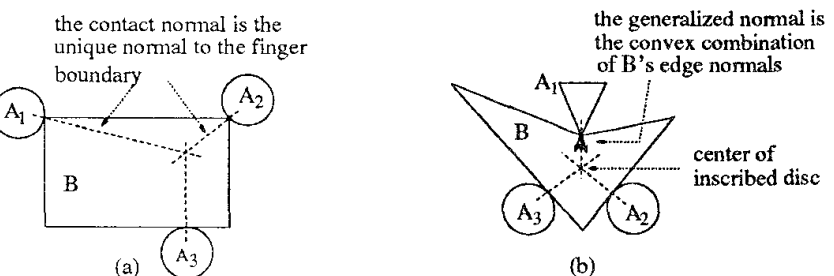

(b)

Figure 7. The contact normal at a (a) convex corner and (b) concave corner

If the contact occurs at a concave corner of $\mathcal{B}, \mathcal{A}_{i}$ must have a "sharp corner" or a vertex at its contact point with $\mathcal{B}$ (Fig. $7(\mathrm{~b})$ ). In this case $\mathcal{B}$ is the local union of two halfplanes, each bounded by one of the edges of $\mathcal{B}$ generating the corner. Thus, if $\mathcal{C B}_{i 1}$ and $\mathcal{C B}_{i 2}$ are the c-obstacles corresponding to the sub-shapes of $\mathcal{B}$, the actual c-obstacle, denoted $\mathcal{C B}_{i}$, is given locally by their union, $\mathcal{C B}_{i}=\mathcal{C B}_{i 1} \cup \mathcal{C B}_{i 2}$. The $1^{\text {st }}$ order free motions of $\mathcal{B}, M_{i}^{1}\left(q_{0}\right)$, is the intersection of the halfspaces of $1^{\text {st }}$ order free motions of $\mathcal{C} \mathcal{B}_{i 1}$ and $\mathcal{C B}_{i 2}$ at $q_{0}$ i.e., $M_{i}^{1}\left(q_{0}\right)$ looks like a "wedge" in $T_{q_{0}} \mathbb{R}^{3}$. The following definition is based on Clarke's notion of generalized gradient [3].

Definition 4 Let a vertex of $\mathcal{A}_{i}$ be in point contact with a concave corner of $\mathcal{B}$ at $y_{i}$. Let $\hat{N}_{i 1}\left(y_{i}\right), \hat{N}_{i 2}\left(y_{i}\right)$ be the unit normals to the edges of $\mathcal{B}$ at $y_{i}$. Then the generalized contact normal at $y_{i}$ is the convex combination $\partial N\left(y_{i}\right) \triangleq \operatorname{co}\left\{\hat{N}_{i 1}\left(y_{i}\right), \hat{N}_{i 2}\left(y_{i}\right)\right\}$ (Fig. $\left.7(\mathrm{~b})\right)$.

We make the reasonable conjecture that $\mathcal{A}_{i}$ can apply contact force along any vector in $\partial N\left(y_{i}\right)$. The conjecture agrees with our intuition, though its rigorous justification is part of ongoing research. As we shall see, at any given equilibrium grasp the direction along which $\mathcal{A}_{i}$ is required to apply a force is uniquely specified.

All the results obtained for the locally maximal inscribed disc of smooth objects also hold for polygons. However, Lemma 3.1 must be rephrased in terms of the generalized contact normal.

Lemma 6.1 ([12]) Let $x$ be a local maximum of $\delta$. Then each $\partial \hat{N}(y)$ for $y \in \mathcal{I}(x)$ contains a vector $\vec{N}(y)$ which points toward $x$, and $0 \in \operatorname{co}\{\vec{N}(y): y \in \mathcal{I}(x)\}$.

\subsection{Immobilization with 3 Convex Fingers}

We now show that any polygon can be immobilized with 3 convex fingers. First consider the case where the locally maximal inscribed disc touches bdy $(\mathcal{B})$ at 3 points.

Proposition 6.2 ([12]) Let $\mathcal{B}$ be a polygon. If a locally maximal inscribed disc touches bdy $(\mathcal{B})$ at three points, $\mathcal{B}$ is completely immobilized by placing 3 fingers in contact with $\mathcal{B}$ at these points.

Sketch of proof: If all the contact points lie in the interior of edges of $\mathcal{B}, \mathcal{B}$ is clearly immobilized by $2^{\text {nd }}$ order effects. Next we argue that if the locally maximal inscribed disc touches bdy $(\mathcal{B})$ at a vertex of $\mathcal{B}$, it must be a concave corner. Thus consider, for instance, the grasp shown in Fig. 7(b). We show that the possible $1^{\text {st }}$ order 
free motions of $\mathcal{B}$ lie in the one-dimensional subspace of instantaneous rotations about the disc center $x$. Then we show that these motions are $1^{s t}$ order penetration with respect to the edges of $\mathcal{B}$ forming the concave corner. Hence $\mathcal{B}$ is immobilized by $1^{\text {st }}$ order effects.

ERROR: syntaxerror's no role in the last proof [12] OFFENDING COMMAND: 245-30zyzowicz et. al [5] that generic polygons can be immobilized by 3 contact STACK : Next consider the case where a locally maximal inscribed disc touches the boundary of $\mathcal{B}$ at two points. We show in [12] that in this case the locally maximal inscribed disc touches $b \operatorname{dy}(\mathcal{B})$ at two parallel edges. The next proposition discusses 3-finger immobilization which starts from two parallel edges.

Proposition 6.3 ([12]) Let $\mathcal{B}$ be a polygon. Let a locally maximal inscribed disc touch bdy $(\mathcal{B})$ at two points. Then there exist thrce points on bdy $(\mathcal{B})$ such that $\mathcal{B}$ is completely immobilized by placing sufficiently flat convex fingers at these points.

The proposition is an adaptation of Prop. 4.4. The only notable difference is that now we rotate $\mathcal{A}_{1}$ and $\mathcal{A}_{2}$ about the respective edge endpoint, rather than sliding them along bdy $(\mathcal{B})$ (Fig. $7(\mathrm{a})$ ). The propositions are summarized in the following theorem.

Theorem 3 All polygons can be immobilized by three frictionless convex fingers, provided that the fingers are sufficiently flat at the contact points.

\subsection{Immobilization with 2 Concave Fingers}

As in the smooth case, we can perform the immobilization at any 2 -point equilibrium grasp, provided that the grasp points do not lie on parallel edges of $\mathcal{B}$.

Proposition 6.4 ([12]) Let $\mathcal{B}$ be a polygon, and let $y_{1}, y_{2} \in \operatorname{bdy}(\mathcal{B})$ determine a 2-point equilibrium grasp. If the edges of $\mathcal{B}$ containing $y_{1}$ and $y_{2}$ are non-parallel, $\mathcal{B}$ is completely immobilized by placing two fingers of suitable curvature at these points.

The following lemma provides a means by which the proposition can be applied to any polygon. The lemma is an adaptation of lemma 5.2.

Lemma 6.5 ([12]) Any polygon $\mathcal{B}$ possesses a 2-point equilibrium grasp such that the grasp points do not lie on parallel edges.

The lemma, together with Proposition 6.4, imply the following theorem.

Theorem 4 All polygons can be immobilized by two suitably curved frictionless fingers.

Note that one contact always occurs at a vertex of $\mathcal{B}$.

\section{Conclusion}

By using curvature effects, we have shown in detail how to immobilize planar smooth and polygonal objects with fewer frictionless fingers or fixtures than were previously thought necessary. By reducing the number of immobilizing fixtures, our results allow greater flexibility in fixture and grasp planning.
Real applications of this work to fixture or grasp planning will invariably involve some amount of friction. However, our focus on immobilization with frictionless fingers reflects a conservative approach - friction always acts to enhance immobilization but it may be arbitrarily small or poorly modeled. Moreover, recent results by Mirtich and Canny [7] suggest that frictional grasps chosen on the basis of frictionless analysis yield optimal disturbance rejection.

There are a number of open issues to be investigated. The proofs of immobility rely on equilibrium grasps which were derived from a locally maximal inscribed disc. It should be emphasized that these immobilizing grasps may not necessarily be the optimalimmobilizing grasps for a given object. The choice of the optimal 2or 3-fingered grasp is a subject of current research. It should also be pointed out that while we have shown how to immobilize objects with fewer fingers, it may be advantageous in some applications to use more fingers than the number prescribed here.

The methods used to prove our results can be extended to $3 \mathrm{D}$ objects. Research in progress shows that any generic smooth or polyhedral 3D object can be immobilized with 4 frictionless convex fingers. This research further suggests that generic smooth or polyhedral 3D objects can be immobilized with 3 suitably concave fingers or fixtures.

\section{References}

[1] M. Berger, Geometry I,II, Springer-Verlag, 1980, NY.

[2] R.C. Brost \& K.Y. Goldberg, A Complete Algorithm for Synthesizing Modular Fixtures for Polygonal Parts, Proc. of ICRA, 1994, pp. 535-542.

[3] F. H. Clarke, Optimization and Nonsmooth Analysis, SIAM Publication, 1990.

[4] F. B. Hazen \& P. K. Wright, Workholding automation: innovations in analysis, design, and planning, Manufacturing Review, 1990, Vol. 3, No. 4, pp. 224-236.

[5] J. Czyzowicz \& I. Stojmenovic \& J. Urrutia, Immobilizing a Polytope, Lecture Notes in Computer Science, Vol. 519,1991 , pp. 214-227.

[6] X. Markenscoff \& L. Ni \& C. H. Papadimitriou, The Geometry of Grasping, Int. J. of Robotics Research, 1990 Vol. 9, No. 1, pp. 61-74

[7] B. Mirtich \& J. F. Canny, Easily Computable Optimum Grasps in 2D and 3D, Proc. of ICRA, 1994, 739-747.

[8] B. Mishra, Workholding, Proc. of IROS, 1991, pp. 53-57.

[9] F. Reuleaux, The Kinematics of Machinery, Macmillan 1876, republished by Dover, 1963, NY.

[10] E. Rimon \& J. W. Burdick, Towards Planning with Force Constraints: On the Mobility of Bodies in Contact, Proc. of ICRA, 1993, pp. 994-1000.

[11] E. Rimon \& J. W. Burdick, Mobility of Bodies in Contact: A New $2^{\text {nd }}$ Order Mobility Index for MultipleFinger Grasps, Proc. of ICRA, 1994, pp. 2329-2335.

[12] E. Rimon \& J. W. Burdick, New Bounds on the Number of Frictionless Fingers Required to Immobilize an Object, Tech Report RMS-94-01, Dept. of ME, Caltech, Sept 1994.

[13] E. Rimon \& J. W. Burdick, A C-space Analysis of Bodies in Contact- $1^{\text {st }}$ Order Mobility and $2^{\text {nd }}$ Order Mobility, Mechanisms and Machine Theory, to appear in spring 1995.

[14] P. Somoff, Zeitschrift fur Mathematic and Physik, 1900, Vol. 45 , pp. 